\title{
An IoT based Urban Areas Air Quality Monitoring Prototype
}

\author{
Martin M. Soto-Cordova ${ }^{1}$, Martha Medina-De-La-Cruz ${ }^{2}$, Anderson Mujaico-Mariano ${ }^{3}$ \\ Universidad de Ciencias y Humanidades \\ Lima, Peru
}

\begin{abstract}
According to the World Health Organization, the most affected places with the presence of polluting gases and particles in suspension are urban areas due to the emissions corresponding to human activities, they have also caused diseases and deaths in millions of people in the world. This paper describes the process of design and implementation of an electronic prototype applying the Internet of Things concept with a cloud storage and processing service. This device has the purpose of monitoring in real-time the air quality through the presence of pollutant gases and PM10 and PM2.5 suspended particles to carry out later studies that contribute to prevention measures in the health care of the population.
\end{abstract}

Keywords-Air pollution; air quality; Arduino; Internet of Things (IoT); cloud service; MQTT; Air Quality Index (AQI); sensors

\section{INTRODUCTION}

Air is a vital resource for all living beings depends mainly on the process of respiration humans. The respiratory frequency of an adult person is 12 to 20 breaths per minute and children 40 to 60 [1]. In the process of respiration, the people are exposed to inhale polluting gases and suspended particles in the air.

According to the World Health Organization (WHO), outdoor air pollution is a major environmental health problem affecting everyone in low-, middle-, and high-income countries. Ambient (outdoor) air pollution in both cities and rural areas was estimated to cause 4.2 million premature deaths worldwide per year in 2016; this mortality is due to exposure to small particulate matter of 2.5 microns or less in diameter (PM2.5), which cause cardiovascular and respiratory disease, and cancers [2, 3]. Likewise, it should be noted that $91 \%$ of countries in the world exceed the guidelines established by WHO. The cases number of deaths increases to the most vulnerable are children under 5 years, pregnant women and older adults. Thus, in 2016, there were 600 million cases of deaths children due to exposure air conditions [4]. Fig. 1 shows the cases of deaths in 2018 of Latin American countries [5]. Therefore, there are world meetings to evaluate this problem and reach agreements between countries to limit air pollution.

There are entities that promote the care of the environment through the practice of cycling (transfer to work centers or study centers), recycling and other activities that reduce emissions in some way by human activity [6], in addition the technology has allowed the development of applications providing innovative ideas such as rapid notification of the existence of natural disasters by emissions that damage the atmosphere, poor conditions of national centers and reserves.

Also, there are monitoring statistics for each region or country made by usually government organizations that show the conditions of air quality. Thus, the Air Quality Index (AQI) value for each pollutant of 100 generally corresponds to an ambient air concentration that equals the level of the shortterm national ambient air quality standard for protection of public health. AQI values at or below 100 are generally thought of as satisfactory. When AQI values are above 100, air quality is unhealthy: at first for certain sensitive groups of people, then for everyone as AQI values get higher. The socalled AQI is divided into six categories that corresponds to a different level of health concern. Furthermore, each category is assigned a certain color, which makes it easy for people to quickly determine whether air quality is reaching levels of health risk in their geographic areas $[7,8]$.

Despite, the people are not informed of these conditions, so their health is at risk when exceeding the permissible ranges and put their lives in danger. In Fig. 2, a cause-effect map related to urban pollution is shown [9]. Therefore, it is necessary to inform the population about these conditions and governorates to take preventive measures. The health of the population should not be affected especially children, elderly, and pregnant women. Apply innovation tools that provide social support to affected populations.

The objective of the research is to monitor air conditions in urban areas using a service in the cloud computing named Adafruit IO by using the device for an analysis that allows spreading this information of the conditions of urban areas at risk. To do this, experimentally, data is collected that belongs to the city of Lima - Peru and is shown.

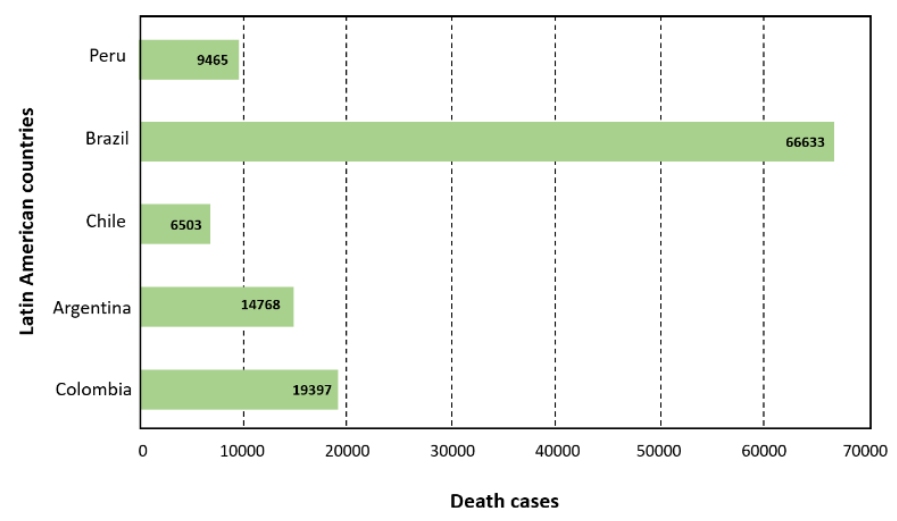

Fig. 1. Cases of Deaths in Latin American Countries. 


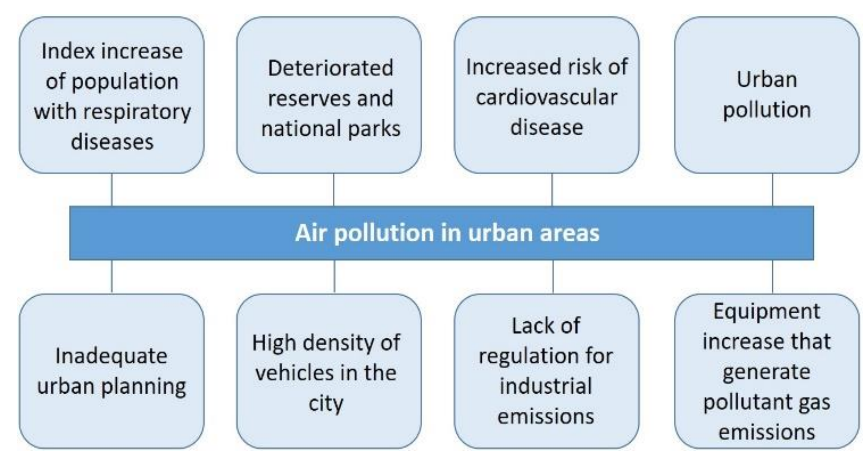

Fig. 2. Causes Effect of Air Pollution.

In what follows, the paper is organized as follows: Section II contains a review of the various proposals for air quality measurement systems, both indoors and outdoors. In Section III, the influence of polluting gases and particles in air suspension on human health will be described. Section IV covers aspects about the procedure for the development of the research project. Finally, the results obtained from the samples collected will be described.

\section{Current Context of the Air Quality Monitoring PLATFORMS}

There are need for real-time air quality monitoring systems for micro, small and medium industries and air pollution in the streets so that timely decisions can be taken to avoid environmental degradation. IoT has been proven one of the effective ways for such systems and when merged with cloud computing provides a revolutionary method of management and analysis of data coming from sensors [10,11].

There are some proposals for indoor monitoring as in [12], an indoor air quality monitoring platform based on IoT technology was developed. It consists of an air quality-sensing device and a web server on cloud computing to monitor indoor air quality. Collected air quality data is transmitted in real time to a web server via LTE mobile network. The IoT device includes pollutant detection sensors, microcontroller and LTE modem, and cloud computing allows visualize indoor air quality data. Likewise, [13] presents an end-to-end indoor air quality monitoring (IAQM) system where the gateway is in charge of processing collected air quality data and its transmission to end-users through a web-server. An adaptation of open-source web application Emoncms was made for live monitoring and long-term storage of the collected IAQM data through sensing technologies, wireless sensor networks (WSNs) and smart mobile.

The author in [14] includes a study where two gaps have been identified: short-term monitoring bias and IAQ datamonitoring solution challenges. The study addresses those gaps by proposing an Internet of Things (IoT) and Distributed Ledger Technologies (DLT)-based IAQ data-monitoring system. The solution helps the penetration of Industrial Internet of Things (IIoT)-based monitoring strategies in the specific case of Occupational Safety Health (OSH).

An air quality monitoring platform under the recommendations of the World Wide Web Consortium (W3C) about the Web of Things (WoT) was proposed in [15]. This system is built based on a WoT capable of exposing its own Thing Description with resources that can serve requests providing measurements of the indoor attached sensors, depending on the underlying hardware and the application protocol selected. On the other hand, a study was carried out on the river Napo located on the border of Ecuador and Peru using a system applied to environmental monitoring to measure the pollution index in different parts of the area. Analyzing the emissions as the existence of toxic waste that damaged the animals that lived in the round of area [16].

An IoT Based Air Quality Monitoring System was proposed in [17]. It considers Arduino based Air Quality Monitoring setup using MQ135 and MQ7 sensors. The data collected by these sensors will be transmitted to the cloud on back end, here it uses Thing speak. Finally, data analysis was done taking into consideration the dataset from experimental tasks. This analysis helps in deeper understanding of the air quality status such that people will be aware of their effects. Furthermore, [18] presents an IoT platform for monitoring indoor air quality. For the implementation of the system the WoT concept is used to create IoT applications and also uses the CoAP protocol to collect data from multiple sensors. This proposal describes the developed platform: sensor hardware, firmware and software to collect the sensors information and to send to the dashboard for visualization.

An IoT based Air and Sound Pollution Monitoring System is presented in [19] to measure the air and noise pollution levels in industrial environment or by using wireless embedded computing system a particular area of interest. It includes IoT with sensing devices connected to the embedded computing system. Also, [20] considers the design and development of an IoT based industrial pollution real time monitoring, from water quality and air pollution and dangerous gas content like ethane and methane. Thus, it includes sensors and micro-controller and the cloud (AWS).

\section{INFLUENCE OF Air Pollution ON HeAlth}

Air pollution affects human health, due to the frequency of exposure to breathing pollutant gases in high concentration were the main effects of allergies and respiratory infections, asthma, bronchitis, premature deaths among others. It is also a factor associated with lung cancer is the cause of 5\% of cases. Fig. 3, on the right side, shows a lung of an older adult where the change in color of the lung is manifested by inhaling particles and gases throughout his life.

Among the main concentrations that harm the health of people are carbon monoxide (CO) gas considered a silent killer belonging to the emissions by vehicles in urban areas especially where there is a high vehicle density or a large number of motorized vehicles with high emissions of these gases due to engine malfunction or wear. Table I shows the emissions of monoxide related by the ratings of the AQI.

In the case of particles in PM10 suspension, which are coarse smaller than 10 microns and that affect the respiratory system entering the pulmonary ducts evaluated in the following equation and evidenced in Table II evaluated in 24 hours:

$\mathrm{I}\left(\mathrm{PM}_{10}\right)=\left[\mathrm{PM}_{10}\right] * 100 / 150$ 


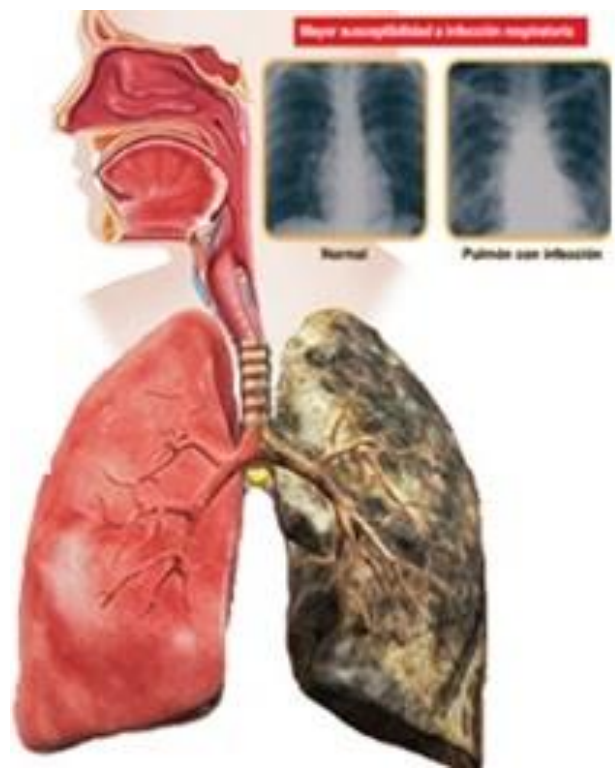

Fig. 3. Effects on the Respiratory System.

TABLE I. RELATED MONOXIDE CONCENTRATIONS THROUGH THE AQI

\begin{tabular}{|l|l|}
\hline AQI Value & Concentration (ppm) \\
\hline $0-50$ & $0.0-4.4$ \\
\hline $51-100$ & $4.5-9.4$ \\
\hline $101-150$ & $9.5-12.4$ \\
\hline $151-200$ & $12.5-15.4$ \\
\hline$>201$ & $15.5-30.4$ \\
\hline
\end{tabular}

TABLE II. PM 10 SusPended Particles Evaluated IN 24 Hours AQI

\begin{tabular}{|l|l|}
\hline AQI Value & Concentration $\left(\boldsymbol{\mu g} / \mathbf{m}^{\mathbf{3}}\right)$ \\
\hline $0-50$ & $0-75$ \\
\hline $51-100$ & $76-150$ \\
\hline $101-150$ & $161-200$ \\
\hline $151-200$ & $250-320$ \\
\hline$>201$ & $>321$ \\
\hline
\end{tabular}

In the case of particles suspended PM10 which are fine, less than 2.5 microns, affect the respiratory system entering the lungs to the lungs, evaluated in the following equation, and shown in Table III:

$\mathrm{I}\left(\mathrm{PM}_{2,5}\right)=\left[\mathrm{PM}_{2,5}\right] * 100 / 25$

Table IV shows the concentration of particles referring to the impact on the health of the person related to the inhalation of $\mathrm{PM}_{10}$.

TABLE III. PM $\quad$ PM.5 $_{2.5}$ SUSPENDEd PARTICles EVAluated IN 24 Hours AQI

\begin{tabular}{|l|l|}
\hline AQI Value & Concentration $\left(\boldsymbol{\mu g} / \mathbf{m}^{\mathbf{3}}\right)$ \\
\hline $0-50$ & $0-12.5$ \\
\hline $51-100$ & $12.6-25$ \\
\hline $101-150$ & $25.1-125$ \\
\hline$>151$ & $>125$ \\
\hline
\end{tabular}

TABLE IV. IMPACT ON HUMAN HEALTH

\begin{tabular}{|l|l|l|}
\hline Con. & Effects & Impact \\
\hline 200 & $\begin{array}{l}\text { Decreased respiratory function and the weakness } \\
\text { of the skin }\end{array}$ & Moderate \\
\hline 250 & $\begin{array}{l}\text { Increase in respiratory and dermatological } \\
\text { diseases }\end{array}$ & Moderate \\
\hline 400 & It affects the entire population & Severe \\
\hline 500 & $\begin{array}{l}\text { Increased mortality in children, pregnant women, } \\
\text { and older adults }\end{array}$ & Very severe \\
\hline
\end{tabular}

The permissible limit according to WHO standards, $\mathrm{PM}_{10}$ is $50 \mathrm{PM}_{10}$ is $50 \mu \mathrm{g} / \mathrm{m}^{3}$ and for $\mathrm{PM}_{2.5}$ is $25 \mu \mathrm{g} / \mathrm{m}^{3}$.

\section{Methodology}

The Internet of Things device is designed to monitor urban areas using a cloud service, which makes it possible for the population to visualize these parameters through access to the Adafruit IO platform. For this reason, the process of development of the IoT device is described:

\section{A. Identification of Variables for Monitoring}

In this part, the presence of gases in urban areas was related to the level of affectation in the vulnerable population, evaluated through the Ministry of Health of Peru (MINSA), relating the inhabitants and their departments. Also, the Ministry of the Environment of Peru (MINAM) and WHO have established levels of concentrations of contaminating particles.

\section{B. Selection of Gas-Related Electrochemical Sensors}

The use of low cost sensors will be used to determine the concentrations of gases in time, so the use of electrochemical sensors was applied, calibrated and analyzed using the equation of the sensitivity curve established by the datasheet. Fig. 4 shows the relationship and the calculation of the equation starting from the value of Rs / Ro for the detection of carbon monoxide (CO) using the MQ-9 sensor.

Fig. 5 shows the relation and calculation of the equation starting from the value of Rs / Ro for smoke detection using the MQ-2 sensor.

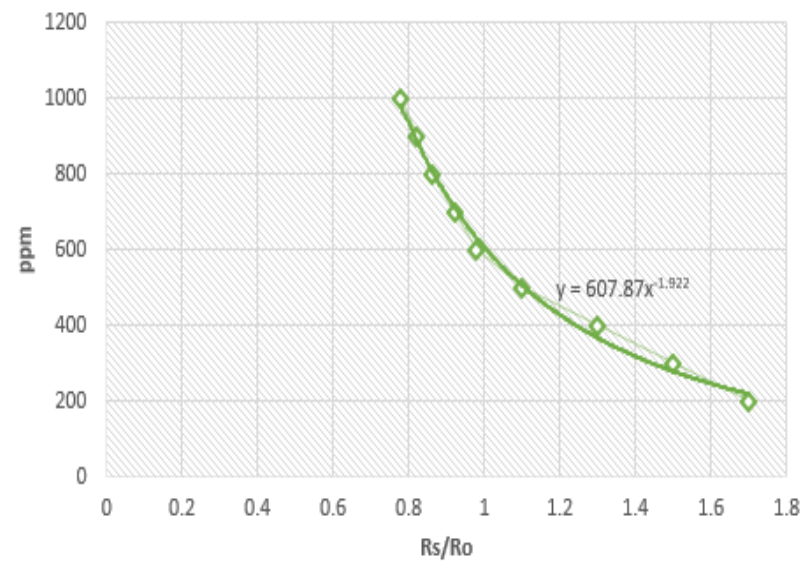

Fig. 4. Sensor Sensitivity Equation MQ 9 for the Detection of Carbon Monoxide (CO). 


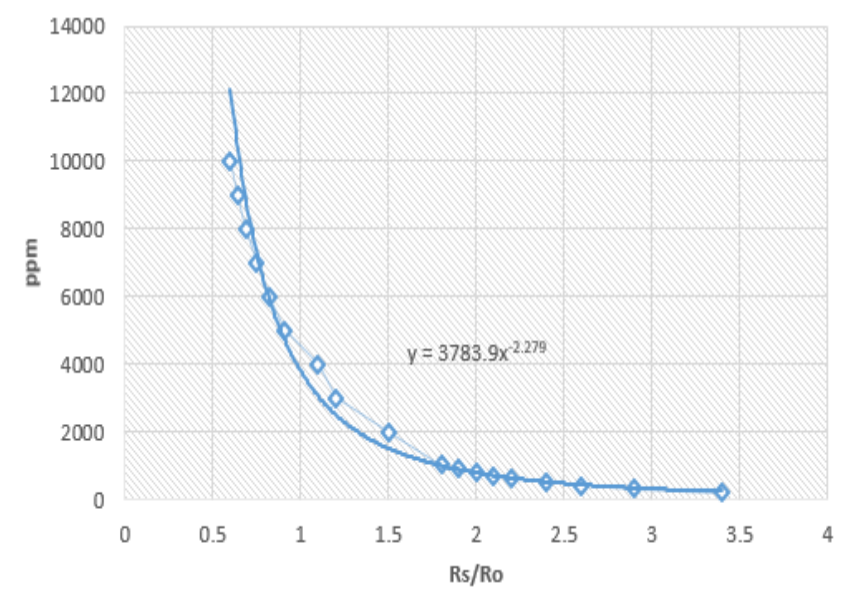

Fig. 5. Sensor Sensitivity Equation MQ 2 for Smoke Detection.

The relationship and calculation of the equation starting from the value of Rs / Ro for the detection of carbon dioxide using the MQ-135 sensor, shown in Fig. 6, is demonstrated.

\section{Design and Implementation of the Internet of Things \\ Device}

For the IoT platform development, the fixed characteristics and requirements are the following:

- Low consumption and low cost;

- Send data to the cloud computing in real-time;

- Allow the visualization of the air pollution conditions of the urban area;

- Small, portable and didactic;

- Monitoring display through an interface;

There are some IoT protocols, such as CoAP and MQTT. Comparatively, CoAP is highly competitive with MQTT, and it includes a mechanism of exploration and observation, but MQTT is much simpler to develop and implement and much more known [21, 22, 23]. Fig. 7 shows that MQTT transaction.

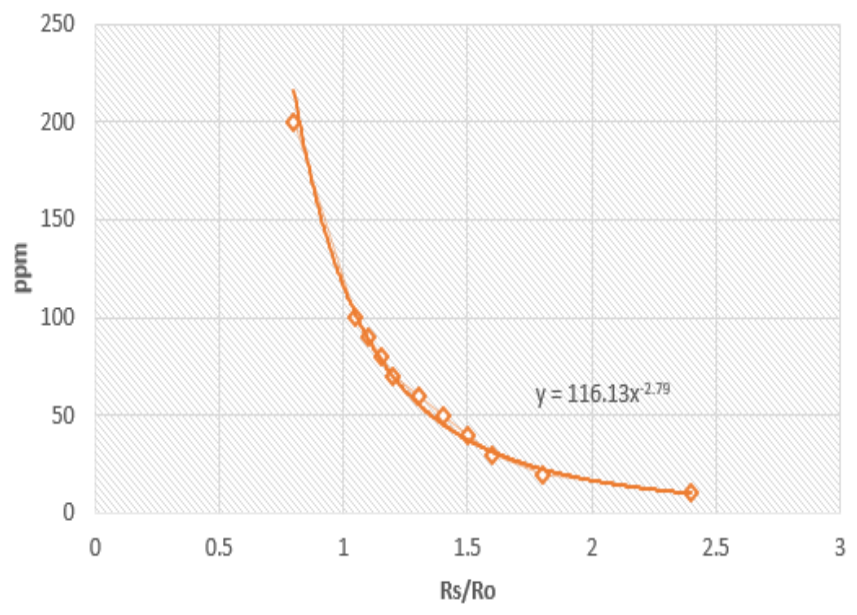

Fig. 6. Sensor Sensitivity Equation MQ 135 for the Detection of Carbon Dioxide $\left(\mathrm{CO}_{2}\right)$.

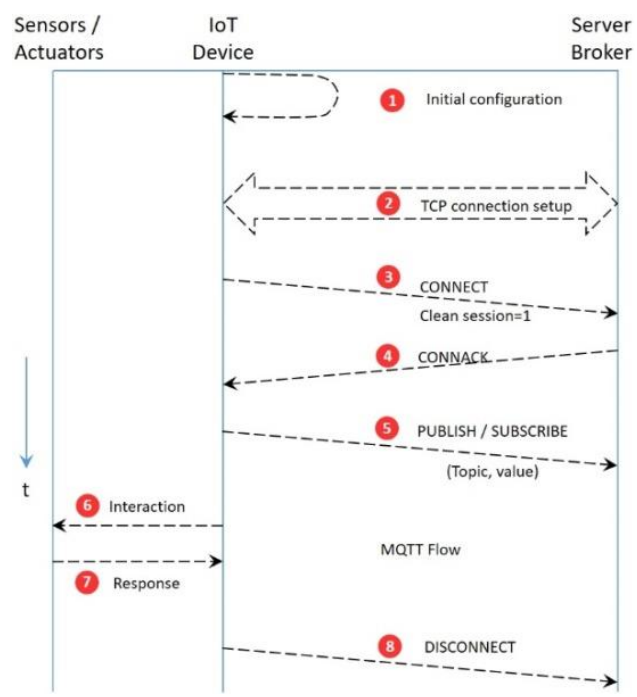

Fig. 7. MQTT Protocol.

The Broker element manages messages and transactions between clients which can be either a subscriber or a publisher. Data is carried in the payload of messages transmitted between clients, mainly a topic and its value. The publisher sends messages to the broker when it has an update message or a periodic message. The broker sends the messages to the subscribers of a specific topic.

Fig. 8 shows an IoT implementation stage. It includes the electrochemical sensors, the humidity, and temperature sensor DHT11, and the dust sensor are connected (obtain data on the density of dust in urban areas). Also, in this process it was decided to work with the Arduino MKR 1000, adapting a program where libraries were used to work with Internet of Things.

Fig. 9 shows the system architecture where IoT device is connected to Adafruit cloud service. It is possible to use Adafruit's IO client API libraries as they include support for MQTT. It allows display data in real-time, online, IoT internet-connected and connect IoT to web services like Twitter, RSS feeds, weather services, etc.

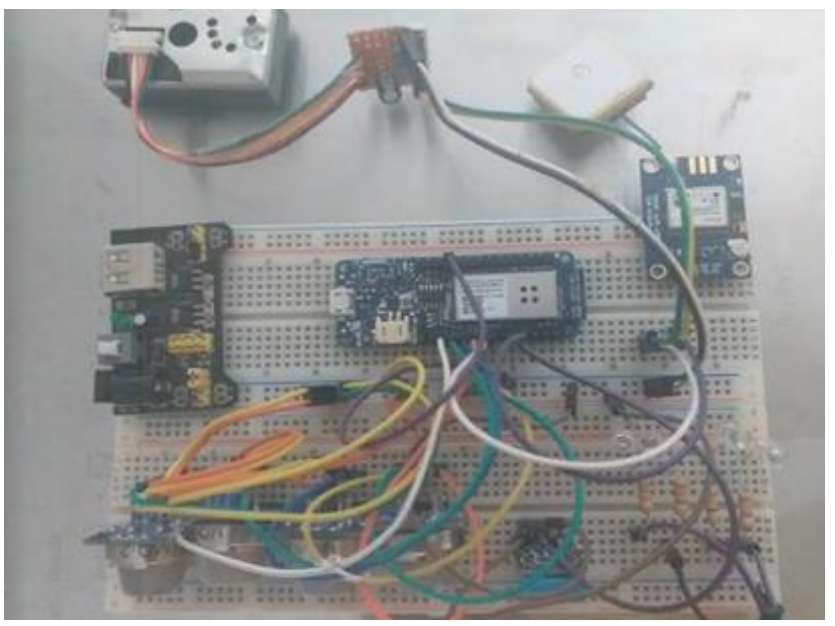

Fig. 8. The Electronic Circuit of the Internet of things Device. 


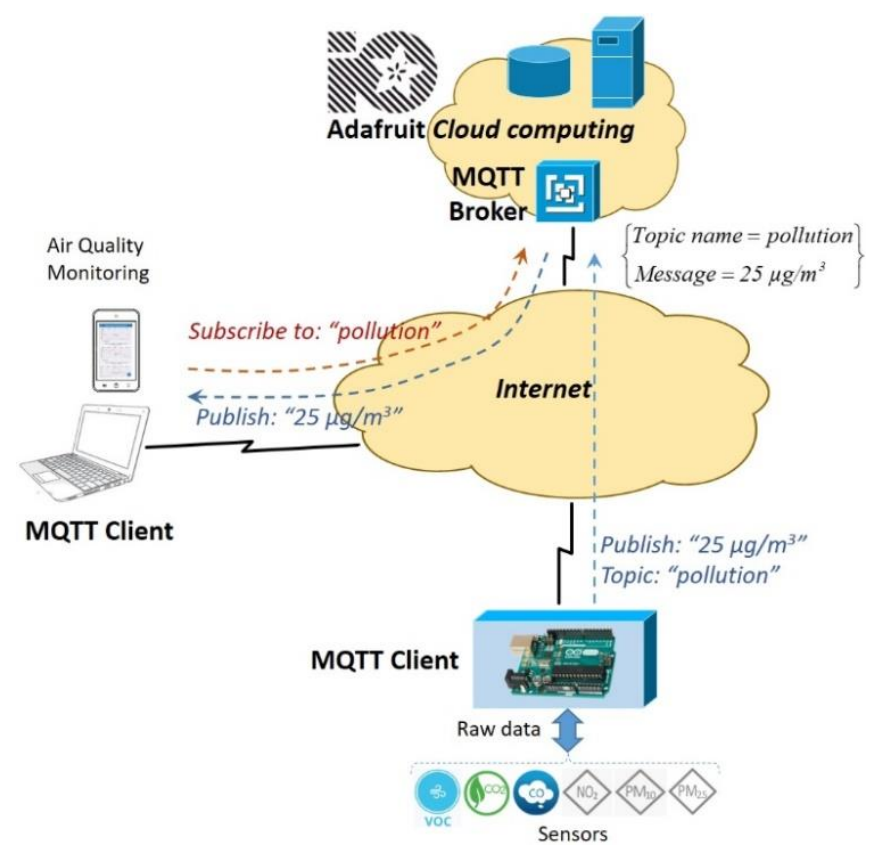

Fig. 9. System Architecture with the use of the Cloud Service.

In this part, the equations in the Arduino IDE were added for the measurement of the electrochemical sensors, to obtain said data in real-time evaluating each message sent by a sensor of 10 seconds. Also, to use the service in the cloud only work with the publication function, which allows visualizing each of the measurements of the sensors in real-time, through the application of an API KEY. Then, Fig. 10 shows some lines of code for reading data.

Adaf ruit_MOTT_Publish humo = Adaf ruit_MOTT_Publish (smqtt, AIO_USERNAME " $/$ feeds/humo");

Adaf ruit MOTT_Publish metano = Adaf ruit MOTT Publish (smqtt, AIO_USERNAME "/feeds/metano"); Adaf ruit_MOTT_Publish monoxido = Adafruit_MOTT_Publish (smqtt, AIO_USERNAME "/feeds/monoxido") Adaf ruit_MOTT_Publish dioxido = Adafruit_MOTT_Publish (smqtt, AIO_USERNAME "/feeds/dioxido"); Adaf ruit_MOTT_Publish hum = Adafruit_MOTT_Publish (smqtt, AIO_USERNAME "/feeds/hum"); Adaf ruit MOTT Publish temp = Adaf ruit MOTT Publish (smqtt, AIO_USERIAME "/feeds/temp"); Adaf ruit_MOTT_Publish temp = Adafruit_MOTT_Publish (smqtt, AIO_USERIAME "/feeds/temp");
Adaf ruit_MOTT_Publish polvo = Adaf ruit_MOTT_Publish (smqtt, AIO_USERIAME "/feeds/polvo"); Adaf ruit_MOTP_Publish densidad = Adafruit_MOTT_Publish (smqte, AIO_USERNAME "/feeds/densidad"

Fig. 10. IDE Programming for Reading Data.

\section{Results}

Fig. 11 shows the monitoring was carried out in different urban areas mainly in areas where industrial activities and avenues are carried out during hours of vehicular congestion. Visualization was carried out using tools from the Adafruit IO board, which varied depending on the concentration of the gas in the area, the measurements were made in varied periods, which are saved by the cloud service.

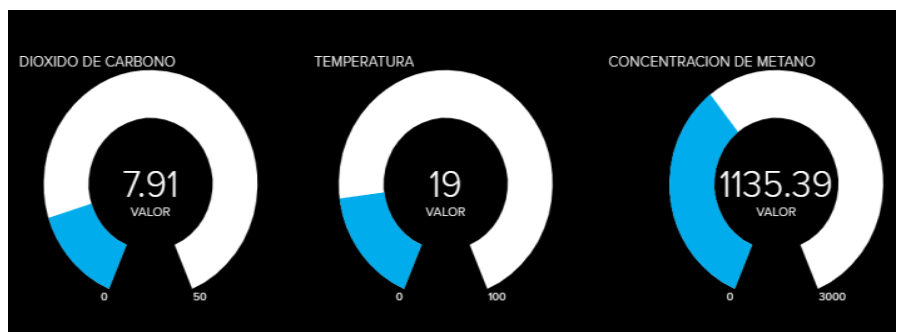

Fig. 11. Visualization of the Existing Concentrations in Ventanilla Area.

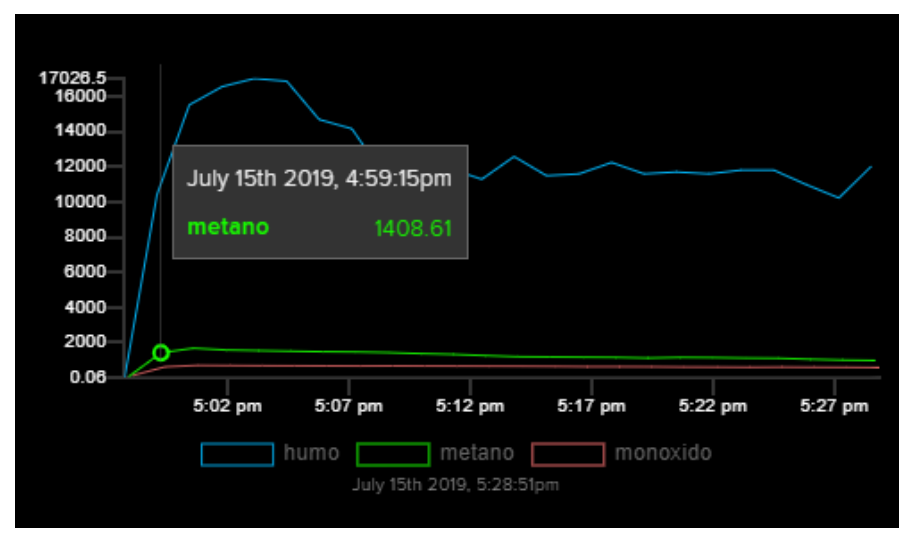

Fig. 12. The Concentration of Measured Gases in Real-Time.

Furthermore, to display several concentrations about time, several gases are configured in the same visualization tool, as shown in Fig. 12.

Through the use of the service, a comparison was made of these concentrations, evaluated in real-time and configured as required by the user, allowing to know the air conditions. Likewise, this information could be shared through a link with other users of the said population to contribute to the reduction of emissions by carrying out activities that could be modified and take care of the area where millions of people live.

\section{CONCLUSION AND FUTURE WORKS}

The Internet of Things has helped to solve tasks in different sectors by managing data and making decisions that could contribute to improving the health of society. For this reason, the use of this device will allow obtaining this information in real-time and publish it through a link so that the population becomes aware of these conditions considering the idea of regulation by monitoring the emissions by the activities human.

Air quality monitoring is a very important task since it would allow adequate policies and control about it in the country, and in this way prevent the affectation of pollution on people's health.

In the future, it is expected to create a network of sensors installed in different areas of Lima, promoting the care of the environment and health care. Also, with the data, this information would be provided to the entities in charge of the monitoring to be disseminated and this information allows knowing the air conditions at the national level.

\section{REFERENCES}

[1] Charilaos Chourpiliadis and Abhishek Bhardwaj, Physiology, Respiratory Rate, StatPearls Publishing, January 28, 2019. [Online]. Available: https://www.ncbi.nlm.nih.gov/books/NBK537306/.

[2] World Health Organization (WHO), Fact sheets: Ambient (outdoor) air pollution, 2 May 2018. [Online]. Available: https://www.who.int/en/ news-room/fact-sheets/detail/ambient-(outdoor)-air-quality-and-health.

[3] World Health Organization (WHO), Ambient air pollution: a global assessment of exposure and burden of disease, 2016.

[4] World Health Organization (WHO), Newsroom: More than $90 \%$ of the world's children breathe toxic air every day, 29 October 2018. [Online]. Available: https://www.who.int/news-room/detail/29-10-2018-morethan-90-of-the-worlds-children-breathe-toxic-air-every-day. 
[5] Expok Comunicación de Sustentabilidad, "Países más contaminados en América Latina - ExpokNews," Comunicacion de Sustentabilidad y RSE, 2019. [Online]. Available: https://www.expoknews.com/ paisesmas-contaminados-en-america-latina/.

[6] A. Budiarto and T. Febriana, "IoT device used for air pollution campaign to encourage cycling habit in inverleith neighborhood," International Conference on Information Management and Technology (ICIMTech), Yogyakarta, pp. 356-360, 2017.

[7] U.S. Environmental Protection Agency (EPA), A Guide to Air Quality and Your Health, EPA-456/F-14-002, February 2014.

[8] U.S. Environmental Protection Agency (EPA), Technical Assistance Document for the Reporting of Daily Air Quality - the Air Quality Index (AQI), EPA-454/B-18-007, September 2018.

[9] Juan M. Rivera Poma, Modelo de identificación de factores contaminantes atmosféricos críticos en Lima-Callao, MSc Thesis, Universidad Nacional Mayor de San Marcos, 2012.

[10] Sumi Neogi et al., "IoT Based Air Quality Monitoring Systems - A Survey," Proceedings of International Conference on Computer Networks, Big Data and IoT (ICCBI), Madurai, Tamil Nadu - India, pp. 752-758, December 19-20, 2018.

[11] Ashish Gupta and Rajesh Kumar, "An IOT Enabled Air Quality Measurement," Indian Journal of Science and Technology, Vol. 11, $\mathrm{N}^{\circ}$ 46, December 2018.

[12] JunHo Jo et al., "Development of an IoT-Based Indoor Air Quality Monitoring Platform," Hindawi - Journal of Sensors, Vol. 2020, January 2020.

[13] Mohieddine Benammar et al., "A Modular IoT Platform for Real-Time Indoor Air Quality Monitoring," MDPI - Sensors, Vol. 18, $\mathrm{N}^{\circ} 581$, 2018.

[14] Shengjing Sun et al., "Indoor Air-Quality Data-Monitoring System: Long-Term Monitoring Benefits," MDPI - Sensors, Vol. 19, $\mathrm{N}^{\circ} 19$, 2019 .
[15] Daniel Ibaseta et al., "An IoT Platform for Indoor Air Quality Monitoring Using the Web of Things," WIT Transactions on Ecology and the Environment, Vol 236, pp. 45-56, 2019.

[16] Joel O. Aragon Valladares, Diseño e implementación de una plataforma de gestión de una red de sensores aplicada a la monitorización de la calidad ambiental en la cuenca del río Napo, Eng. Thesis, Pontificia Universidad Católica del Perú, 2014.

[17] Kinnera Bharath Kumar Sai, Subhaditya Mukherjee and H. Parveen Sultana, "Low Cost IoT Based Air Quality Monitoring Setup Using Arduino and MQ Series Sensors With Dataset Analysis," Procedia Computer Science, Vol 165, pp. 322-327, 2019.

[18] J. Esquiagola et al., "Monitoring Indoor Air Quality by using IoT Technology," IEEE XXV International Conference on Electronics, Electrical Engineering and Computing (INTERCON), Aug. 2018

[19] Pradyumna Bapat et al., "IoT based Air and Sound Pollution Monitoring System," International Journal of Research and Analytical Reviews (IJRAR), Vol. 6, $\mathrm{N}^{\circ}$ 2, pp. 383-387, April-June 2019.

[20] Raghava M S et al., "IoT based Industrial Pollution Monitoring," Research Journal of Engineering and Technology (IRJET), Vol. 6, $\mathrm{N}^{\circ} 5$, pp. 5893-5899, May 2019.

[21] Khalid Aloufi, "6LoWPAN Stack Model Configuration for IoT Streaming Data Transmission over CoAP," International Journal of Communication Networks and Information Security (IJCNIS), Vol. 11, №2, pp. 304-311, August 2019.

[22] T. Yokotani and Y. Sasaki. "Transfer protocols of tiny data blocks in iot and their performance evaluation," IEEE 3rd World Forum on Internet of Things (WF-IoT), pp. 54-57, 2016.

[23] T. Yokotani and Y. Sasaki, "Comparison with HTTP and MQTT on Required Network Resources for IoT," International Conference on Control, Electronics, Renewable Energy and Communications (ICCEREC), pp. 1-6, 2016. 